\title{
Einfluss der Bildung der Frau auf das Zweitgeburtsverhalten in Polen während der sozio- ökonomischen Transformation ${ }^{1}$
}

\section{Impact of educational attainment on the transition to the second child in post-socialistic Poland}

\section{Zusammenfassung:}

In Polen wurde nach dem Systemumbruch im Jahr 1989 ein starker Geburteneinbruch beobachtet, der nahezu parallel von einer Bildungsexpansion begleitet wurde. Der schnell steigende Anteil junger hoch gebildeter Frauen sollte sich stark auf die zukünftige Fertilitätsentwicklung Polens auswirken, denn Akademikerinnen bekommen nicht nur später, sondern auch weniger Kinder. Die Studie lenkt den Fokus auf das Zweitgeburtsverhalten, da die Geburt des ersten Kindes in Polen weiterhin als universell gilt. Die empirischen Ergebnisse auf Basis des Employment, Family and Education Surveys (2006) zeigen eine mit steigendem Bildungsniveau signifikant sinkende Zweitgeburtenrate. Hochschulabsolventinnen sind in Polen mit hohen Opportunitätskosten konfrontiert, die nur eingeschränkt von den geltenden familienpolitischen Maßnahmen und Arbeitsmarktregulierungen minimiert werden.

Schlagwörter: Polen, Fertilität, zweites Kind, Bildung

\begin{abstract}
:
Since the collapse of state socialism in Poland in 1989, a rapid decline in fertility has been observed in parallel with educational expansion. Polish women with university degree tend to postpone their childbearing, and often have fewer children than their less educated counterparts. Thus, an increase in the number of highly educated women may have an impact on the future fertility development of a country. The birth of a first child is fairly universal in women's lives in Poland, hence, this study focuses on the transition to the second child. Using the Employment, Family and Educational Survey (2006), several piecewise exponential models were estimated. The analyses reveal a strong negative effect of women's education on the second birth risk. We conclude that Polish women with university degree are exposed to higher opportunity costs, and these are not addressed adequately by existing family policies and labour market regulations related to working mothers.
\end{abstract}

Key words: Poland, fertility, second birth, education

1 Das Manuskript basiert auf der Masterarbeit eingereicht an der Universität Rostock im August 2009. Die vorgestellten Ergebnisse wurden auch in einer Postersession auf der Konferenz Population Association of America (PAA) 2011 Annual Meeting, Washington DC, USA, 31.03-02.04.2011 präsentiert. 


\section{Einleitung}

Die ehemaligen Ostblockstaaten verzeichneten nach dem Systemumbruch um das Jahr 1990 einen dramatischen Geburtenrückgang, der von deutlichen Veränderungen im altersund paritätsspezifischen Muster begleitet wurde (Council of Europe 2006). ${ }^{2}$ Die Totalen Fertilitätsraten (TFR) sanken lediglich innerhalb eines Jahrzehntes drastisch - von meistens über 1,8 Kinder je Frau zu Beginn der Transformationsjahre bis zu unter die „lowest low"-Grenze (TFR $\leq 1,3$; nach Kohler et al. (2002)). In der ganzen Region wurde dabei ein gestiegenes Alter beim ersten Kind, ein sinkender Anteil der Geburten der dritten und höheren Ordnung und eine zunehmende Kinderlosigkeit beobachtet (Frejka/Sobotka 2008; Sobotka 2004: Kap. 5; Sobotka et al. 2005). Neuerdings häuft sich auch die Befürchtung einer Erosion des in diesem Teil Europas vorherrschenden Zwei-Kinder-Familienmodels (Frejka 2008a; Frejka/Sardon 2007; Kreyenfeld 2008), die durchaus schwerwiegende Folgen für die künftige Bevölkerungsentwicklung Osteuropas nach sich ziehen könnte (Lutz et al. 2006).

Der beobachtete familiendemographische Wandel wird hauptsächlich auf tiefgreifende sozio-ökonomische Veränderungen zurückgeführt, die mit dem Regimewechsel und dem Übergang von der zentralen Planwirtschaft zur freien Marktwirtschaft einhergingen (Frejka 2008b; Kotowska et al. 2008; Potančoková et al. 2008; Sobotka et al. 2008; Spéder/Kamarás 2008; Witte/Wagner 1995). Daraufhin nahm auch die Bedeutung des Humankapitals für die persönlichen Lebensverhältnisse und Berufschancen enorm zu. Seit dem Beginn der 1990er Jahre wird in den meisten ehemaligen Ostblockstaaten eine vor allem von Frauen jüngerer Jahrgänge vorangetriebene Bildungsexpansion beobachtet (z.B. Kotowska et al. 2008; Potančoková et al. 2008; Sobotka et al. 2008; Spéder/Kamarás 2008). Ein rasant steigender Anteil der hoch gebildeten Frauen dürfte dabei Implikationen für die zukünftige Fertilitätsentwicklung dieser Region haben. Hochschulabsolventinnen unterscheiden sich in ihrem generativen Verhalten deutlich von Frauen niedrigerer Bildungsgruppen; sie bekommen ihr erstes Kind meistens später und entscheiden sich häufig für kleinere Familien (z.B. Blossfeld/Huinink 1991; Lappegård/Rønsen 2005; Liefbroer/Corijn 1999). Zahlreiche Studien wiesen einen negativen Bildungseffekt auf den Eintritt in die Mutterschaft auch in den ehemaligen Ostblockstaaten nach (z.B. Kantorová 2004; Koytcheva 2006; Matysiak 2009a, 2009b; Matysiak/Vignoli 2009). Der Einfluss des Schulabschlusses auf das Zweitgeburtsverhalten ist jedoch weniger eindeutig. Während in Westeuropa und Skandinavien ein positiver Bildungsgradient dokumentiert wurde (z.B. Gerster et al. 2007; Hoem/Hoem 1989; Köppen 2006; Kreyenfeld/Zabel 2005; Oláh 1996), ist das in den ehemaligen Ostblockstaaten nur in Estland (Klesment/ Puur 2010) und Tschechien (Št'astná 2009) der Fall. In der Mehrheit der postsozialistischen Länder nimmt das Risiko einer zweiten Geburt mit steigendem Schulabschluss ab: Rumänien (Mureşan 2007), Russland (Rieck 2006), Ukraine (Perelli-Harris 2008), Bulgarien (Koytcheva 2006) und Ungarn (Oláh 2003). Vor dem Hintergrund unterschiedlicher

2 Einen detaillierten Überblick über die familiendemographischen, sozio-ökonomischen und familienpolitischen Veränderungen in den einzelnen ehemaligen Ostblockstaaten liefert der Sammelband der Zeitschrift Demographic Research (2008) mit dem Titel „Special collection 7: Childbearing trends and policies in Europe". 
Ergebnisse und für unser besseres Verständnis des Fertilitätsrückgangs in Osteuropa scheint eine weitere Untersuchung des Zusammenhangs zwischen Schulabschluss und Familienerweiterung in einem bisher unerforschten sozio-ökonomischen und kulturellen Kontext von besonderer Relevanz.

Das Ziel der vorliegenden Studie ist demnach, den Einfluss der Bildung auf den Übergang zum zweiten Kind im postsozialistischen Polen zu ermitteln. $\mathrm{Zu}$ dem Effekt des Schulabschlusses auf das Zweitgeburtsverhalten liegen, unseres Wissens nach, noch keine empirischen Untersuchungen vor. Unsere Überlegungen beruhen auf dem ökonomischen Ansatz der Fertilität (z.B. Becker 1963, 1993), auf dessen Grundlage die Rolle der Vereinbarkeit von Kind und Beruf erörtert wird. Außerdem wird die Auswirkung der Bildung der Frau auf Familiengründung und -erweiterung aus der Lebenslaufperspektive betrachtet. Nachdem der polnische Kontext beleuchtet wird, folgt die Formulierung der Forschungshypothesen, deren Gültigkeit auf Basis des Employment, Family and Education Survey aus dem Jahr 2006 empirisch geprüft wird. Die empirische Analyse erfolgt mithilfe von Methoden und Techniken der Ereignisdatenanalyse.

\section{Theoretische Vorüberlegungen}

Die theoretischen Überlegungen zum Einfluss der Bildung der Frau auf die Fertilitätsentscheidung basieren auf dem ökonomischen Ansatz der Fertilität (z.B. Becker 1965, 1993). Dieser sieht Frauen als rational handelnde, nutzenmaximierende Akteure, die vor dem Hintergrund konstanter Präferenzen und gegebener Budgetrestriktionen bestrebt sind, ihre verfügbare Zeit zwischen den Haushalts- und Arbeitsmarktaktivitäten optimal aufzuteilen. Der Wert der Zeit einer Frau wird dabei mit dem Stundenlohn gemessen, zu dem sie auf dem Arbeitsmarkt beschäftigt wird. Je höher das Bildungsniveau einer Frau ist, umso besser sind ihre Arbeitsmarktchancen und damit auch das Verdienstpotenzial (Mincer 1974; Mincer/Polachek 1974). Hoch gebildete Frauen sollen daher einen höheren Anreiz haben, einer Erwerbsarbeit nachzugehen.

Die mit zunehmendem Schulabschluss ebenfalls steigenden Löhne wirken sich zweierlei - über den Einkommens- und den Substitutionseffekt - auf die Fertilitätsentscheidungen einer Frau aus (z.B. Ermisch 2003: 114-117; Matysiak 2009b: 58f.). Der Einkommenseffekt unterstellt einen positiven Einfluss steigender Bildung der Frau auf die Familiengröße. Höhere Einkünfte verhelfen einerseits die anfallenden direkten Kinderkosten (Ernährung, Bekleidung, Bildung etc.) abzudecken. Andererseits vermögen sie die entstandenen Zeitkosten der Kindererziehung zu reduzieren, wie etwa durch einfachere Finanzierung der kostenpflichtigen außerfamiliären Kinderbetreuungsmöglichkeiten. Der Substitutionseffekt wirkt in die entgegengesetzte Richtung und spiegelt den Zeitkonflikt zwischen Erwerbstätigkeit der Frau und häuslicher Kinderbetreuung wider. Die Zeit, die eine Frau für Erziehung der Kinder und Verrichtung der Haushaltsarbeiten aufbringt, könnte der Erwerbsarbeit gewidmet werden. Mit steigendem Bildungsniveau erhöhen sich das zu erwartende Einkommen und damit auch die Opportunitätskosten der Kindererziehung. Da Frauen aus ökonomischer Sicht rational handeln, versuchen sie diese Kosten zu minimieren, indem sie sich für kleinere Familien entscheiden. Der Substitutionseffekt impliziert folglich einen negativen Einfluss des höheren Schulabschlusses der Frau auf ihre Kinderzahl. 
Die endgültige Entscheidung für oder gegen eine (weitere) Mutterschaft wird durch das Zusammenspiel zwischen dem Substitutions- und dem Einkommenseffekt bestimmt. Beide Effekte treten gleichzeitig auf und beeinflussen gegenläufig die Familienplanung der Frau. Ob eine Familie erweitert wird, hängt schließlich davon ab, welcher der Effekte dominiert. Das Zusammenspiel beider Effekte wird dabei weitgehend durch die gesellschaftlichen Gegebenheiten bestimmt, in denen die Fertilitätsentscheidungen getroffen werden (Matysiak 2009b). Hierbei sind die länderspezifischen sozialen, ökonomischen, politischen und kulturellen Rahmenbedingungen gemeint, die die Entscheidung über ein (weiteres) Kind begünstigen oder hindern können (z.B. Gornick et al. 1998; Liefbroer/ Corijn 1999; Matysiak 2009b; Muszyńska 2004, 2007; Rindfuss et al. 2003). Folglich kann der Einfluss der Bildung auf Familiengründung und Erweiterung zwischen den Ländern stark variieren (Blossfeld 1995).

Das Ausmaß des Substitutionseffekts lässt sich über die Höhe der Opportunitätskosten bestimmen. Da Betreuung der Kinder und Erwerbsarbeit der Frau als konkurrierende Aktivitäten um die weiblichen Zeitressourcen gelten, fallen die Opportunitätskosten der Kindererziehung mit zunehmendem Schulabschluss umso geringer aus, je besser sich diese beiden Tätigkeiten miteinander vereinbaren lassen (Liefbroer/Corijn 1999). Eine zentrale Bedeutung wird hier den familien- und arbeitsmarktpolitischen Regulierungen zur Unterstützung der Mutterschaft und Frauenerwerbstätigkeit zugeschrieben (Matysiak 2009b: 61; Neyer 2006). Dazu gehören etwa die Bestimmungen zum Mutterschafts- und Erziehungsurlaub, die Versorgung mit bedarfsgerechter Kinderbetreuung, sowie Regulierungen $\mathrm{zu}$ den Arbeitszeiten, vor allem deren Umfang (Voll- und Teilzeit), und dem (Wieder-)Einstieg der Frau in das Berufsleben. Zu beachten sind zudem auch alle Instrumente, die sich auf das Haushaltsbudget und somit auch auf das Ausmaß des Einkommenseffekts auswirken. Als solche gelten diverse Leistungen, die auf Kompensierung der direkten Kinderkosten abzielen. $\mathrm{Zu}$ nennen sind vor allem familienbezogene monetäre Transfers (Mutterschafts-, Erziehungsgeld, Familienbeihilfen etc.), steuerliche Vergünstigungen für Familien mit Kindern sowie Zuschüsse zu Dienstleistungen, etwa für Gesundheitsvorsorge, Bildung oder öffentliche Verkehrsmittel (Wóycicka 2005: 80).

Die ökonomische Theorie der Fertilität thematisiert den Einfluss der Bildung der Frau auf die Familiengröße, jedoch nicht den Zeitpunkt und den Abstand zwischen den Geburten. Dabei wird jede familiäre Entscheidung, mit all ihren Konsequenzen für die zukünftigen Handlungsmöglichkeiten, in einem bestimmten Moment des Lebens getroffen. Frauen haben, von sozialen Alterspräferenzen abgesehen, ein relativ breites Zeitfenster, das lediglich von der biologischen Grenze einschränkt wird, um ihren Kinderwunsch zu realisieren. Die reproduktive Phase fällt allerdings mit dem Lebensabschnitt zusammen, in dem die Integration in den Arbeitsmarkt stattfindet (Schmitt 2007: 3). Familiäre Entscheidungen einer Frau und ihr berufliches Engagement stehen entsprechend in Wechselwirkung zueinander und üben einen strukturierenden Einfluss auf den Lebenslauf der Frau aus (ibd.: 4).

Eine erhöhte Bildungspartizipation der Frau wirkt sich wesentlich auf das Timing der Familiengründung und Erweiterung aus. Akademikerinnen sind für gewöhnlich älter bei Geburt des ersten Kindes als Frauen mit niedrigerem Schulabschluss. Längeres Verweilen in dem Bildungssystem trägt unmittelbar zum zeitlichen Hinauszögern von familiären Entscheidungen bei (z.B. Blossfeld/Huinink 1991; Klein/Lauterbach 1994; Lappegård/ 
Rønsen 2005). Sowohl das Hochschulstudium als auch die Mutterschaft sind mit hohen Zeitanforderungen verbunden, wodurch sich die beiden Lebensbereiche nur schwer miteinander vereinbaren lassen. Die während der Ausbildung meistens noch unsichere finanzielle Lage sowie die normativen Erwartungen erst Schulabschluss, dann Kinder (Blossfeld/Huinink 1991) sprechen weiterhin für einen Aufschub der Familiengründung. Schließlich sollen die langfristigen Opportunitätskosten eines familienbedingten Ausbildungsabbruchs deutlich höher als die der späteren Erwerbsunterbrechung ausfallen (Klein/Lauterbach 1994).

Sobald das Studium abgeschlossen ist, scheint es rational, die erworbenen Bildungsressourcen in berufliche Statuspositionen zu übertragen (Schmitt 2007: 4). Da das Einkommensprofil der Hochschulabsolventinnen steiler als das der Frauen mit einem niedrigeren Schulabschluss verläuft, sind die Opportunitätskosten der Kindererziehung in den ersten Berufsjahren besonders hoch (Liefbroer/Corijn 1999: 54). Eine Unterbrechung in der Erwerbskarriere zugunsten häuslicher Kinderbetreuung bedeutet nicht nur entgangenes Einkommen, sondern auch fehlende Investitionen bis hin zum Abbau des Humankapitals. Letzteres kann sich heute, in den Zeiten des schnellen, technologischen Fortschritts, gerade bei einem langen Ausstieg aus dem Berufsleben negativ auf die zukünftigen Karrierechancen und das Erwerbspotenzial der Frau auswirken (z.B. Aisenbrey et al. 2009; Albrecht et al. 1999; Mincer/Ofek 1982). Die Familiengründung wird daher häufig bis zur Etablierung auf dem Arbeitsmarkt hinausgezögert. Ein Aufschub der Mutterschaft bedeutet zugleich eine Verkürzung der Zeitspanne, in der die Realisierung des Kinderwunsches möglich ist. Die Akademikerinnen können entsprechend bestrebt sein, ihre Kinder zeitlich eng nacheinander zu bekommen. In der Literatur wird in solchem Fall von einem „Time-Squeeze“ gesprochen (Kreyenfeld 2002). Eine Minimierung des Geburtenabstandes dürfte sich in weitestem Sinne auch positiv auf die Erwerbskarriere der Frauen auswirken, da sie eine schnelle Rückkehr in das Erwerbsleben und daher auch die Reduzierung der Opportunitätskosten ermöglicht (NíBhrolcháin 1986a, b).

\section{Polen nach dem Systemumbruch 1989}

\subsection{Entwicklung der Fertilität}

Mit dem Systemumbruch im Jahr 1989 ging in Polen die TFR von 2,07 dramatisch auf 1,22 im Jahr 2003 zurück und stabilisierte sich in den letzten Jahren bei etwa 1,3 Kindern je Frau. ${ }^{3}$ Das Durchschnittsalter bei Geburt des ersten Kindes stieg gleichzeitig von 23,6 auf 25,9 Jahre an, sowie von 26,8 auf 28,1 Jahre bei Geburt aller Kinder. Zudem ist der Anteil der dritten und vierten Geburten an allen Geburten seit dem Jahr 1989 von jeweils $17 \%$ auf $10 \%$ und von 12\% auf knapp 6\% im Jahr 2007 gesunken. Interessanterweise erweist sich der Kinderwunsch in Polen, trotz des Rückgangs der Fertilität, als nahezu uni-

3 Die hier verwendeten Daten für Polen stammen hauptsächlich von den Datenbank OECD.Stat Exracts und ILO LABORSTA, sowie vom Zentralen Statistischen Amt in Warschau (auf Polnisch: Główny Urząd Statystyczny, GUS). Zusätzlich verwendete Datenquellen werden ggf. separat angegeben. 
versal. Wenn auch die Kinderlosigkeit in den jüngeren Kohorten von 6,9\% für die Kohorte 1938 auf 15\% für den Jahrgang 1966 anstieg (Kotowska et al. 2008: 807), beabsichtigten im Eurobarometer Survey aus dem Jahr 2006 lediglich 1,6\% der 15 bis 39-jährigen kinderlosen Polinnen keine Mutterschaft zu realisieren (Testa 2006).

\subsection{Sozio-ökonomische Rahmenbedingungen}

Der dramatische Geburtenrückgang und der damit einhergehende alters- und paritätsspezifische Wandel des Fertilitätsmusters polnischer Frauen werden, wie in allen ehemaligen Ostblockstaaten, hauptsächlich auf die tiefgreifenden sozio-ökonomischen und institutionellen Veränderungen zurückgeführt, die der Systemumbruch in 1989 mit sich brachte (Kotowska et al. 2008; Kotowska 1999). Anfang der 1990er Jahre wurden zahlreiche ökonomische Reformen eingeleitet, die eine weitgehende Liberalisierung der polnischen Wirtschaft (etwa Freisetzung der Preise, Privatisierung und Restrukturierung staatlicher Unternehmen, Anpassung der Beschäftigungsstruktur an marktwirtschaftliche Kriterien der Produktionsauslastung) beabsichtigten. Die ersten Transformationsjahre Polens waren von einem starken Rückgang der Beschäftigung und des Bruttoinlandsproduktes gekennzeichnet. Zugleich trat massive Arbeitslosigkeit (1993: 14,4\%), ein im Sozialismus unbekanntes Phänomen, auf. In den folgenden Jahren wurden eine kurzzeitige Verbesserung der wirtschaftlichen Lage und eine leichte Entspannung auf dem Arbeitsmarkt beobachtet. Seit dem Jahr 1998 verlangsamte sich jedoch das Wirtschaftswachstum und die Arbeits-

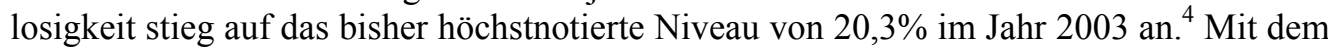
EU-Beitritt Polens im Mai 2004 setzte eine anhaltende Hochkonjunktur ein. Die Erwerbsquote blieb auf einem relativ konstanten Niveau von durchschnittlich 64,1\% (vgl. 1988: $71,5 \%$ ), die Arbeitslosenquote sank kontinuierlich auf einen Wert von unter $10 \%$ und die jährliche Wachstumsrate des BIP stieg bis zum Jahr 2007 auf ca. 6\% an.

Der Systemumbruch eröffnete bisher unbekannte Chancen hinsichtlich individueller Wahlfreiheiten, Lebensgestaltung und Konsummöglichkeiten, jedoch nicht für alle in gleichem Maße (Kotowska 2004). Ökonomische und soziale Ungleichheiten spiegelten sich in der verstärkten Polarisierung der Gesellschaft, steigender Armut und sozialer Ausgrenzung bestimmter Gruppen wider (Kotowska et al. 2008: 822f.). Aufgrund erschwerter Partizipation auf dem Arbeitsmarkt sowie der Abkehr des Staates von seiner bisher übergeordneten Funktion der Absicherung persönlicher Lebensverhältnisse, hängt nun die finanzielle Lage privater Haushalte vorwiegend von den Ressourcen ihrer Mitglieder ab. Die insgesamt gestiegene Eigenverantwortung für das materielle Wohlergehen einerseits und die wachsende Nachfrage nach gut qualifizierten Arbeitskräften andererseits, erhöhten dabei die Bedeutung des eigenen Humankapitals, was eine fortschreitende Bildungs-

4 Der Verschlechterung der Situation auf dem Arbeitsmarkt zu Beginn des 21. Jahrhunderts lagen einerseits der weitere Stellenabbau infolge anhaltender Restrukturierungs- und Privatisierungsprozesse und andererseits die aus steigendem Wettbewerb und Druck nach mehr Effizienz und Produktivität resultierenden Veränderungen der Beschäftigungsstruktur zugrunde. 
expansion auslöste. ${ }^{5}$ Diese wurde dabei hauptsächlich von den Frauen jüngerer Geburtsjahrgänge vorangetrieben; der Anteil der Akademikerinnen in der Altersgruppe der 25bis 34-Jährigen erhöhte sich seit dem Systemumbruch um den Faktor 3,6 und lag im Jahr 2005 bei knapp 31\%. ${ }^{6}$

Die hoch gebildeten Polinnen unterscheiden sich dabei in ihrem generativen Verhalten wesentlich von Frauen anderer Bildungsgruppen. Sie verweilen deutlich länger in der Ausbildungsphase, sind aber - ähnlich wie die geringer qualifizierten Frauen - bestrebt, bereits vor der ersten Mutterschaft im Arbeitsmarkt vollkommen integriert zu sein (Matysiak 2009b; Matysiak/Vignoli 2009). Im Jahr 2007 lag das Medianalter der Akademikerinnen bei der ersten Geburt bei 28,1 und das der Frauen aller Bildungsgruppen bei 25,8 Jahren (GUS 2008b). Die Betrachtung der jährlichen Zahl der Lebendgeborenen nach durchschnittlicher Geburtenordnung verdeutlicht zudem, dass die hoch gebildeten Polinnen nicht nur später sondern auch weniger Kinder in ihrem Leben bekommen. ${ }^{7}$

\subsection{Frauenerwerbstätigkeit}

In den Zeiten des Sozialismus war die Frauenerwerbstätigkeit ideologisch angestrebt, gemäß Vollbeschäftigungspolitik staatlich gefördert und für das Haushaltsbudget angesichts der Politik der niedrigen Löhne notwendig. Die strukturellen Veränderungen auf dem Arbeitsmarkt nach dem Jahr 1989 beeinträchtigten erheblich das Erwerbsleben der Frauen. Die Frauenerwerbsquote ging in den Transformationsjahren kontinuierlich von 64\% in 1988 auf 50,6\% im Jahr 2007 zurück. Ein höherer Schulabschluss reduzierte dabei in jedem Alter das Risiko von Arbeitslosigkeit betroffen zu werden und verbesserte die Chancen der Frau eine Vollzeitbeschäftigung, und dies sogar unbefristet, aufzunehmen ${ }^{8}$ (Matysiak 2007).

Die höchste Erwerbsbeteiligung der Polinnen fällt mit der Phase verstärkter familiärer Entscheidungen, d.h. mit dem Alter 25-44, zusammen (Kotowska/Sztanderska 2007; Sztanderska 2005). Die Beschäftigung der Frau stellt eine sehr wichtige Voraussetzungen für eine (weitere) Mutterschaft dar (Matysiak 2009a; Matysiak/Vignoli 2009). Nach der Geburt des Kindes sinkt die Erwerbsbeteiligung der Frauen jedoch rapide ab. Zahlreiche empirische Untersuchungen bestätigen einen starken negativen Einfluss von Kleinkindern auf die Müttererwerbstätigkeit (Matysiak 2005, 2009a; Matysiak/Steinmetz 2008). Die Polinnen ziehen sich vorerst aus dem Berufsleben zurück, um sich der häuslichen Kinderbetreuung zu widmen. Wenn das Kind älter ist, versuchen sie in das Berufsleben zurückzukehren, stoßen aber auf große Schwierigkeiten; sie sind oft von Arbeitslosigkeit betroffen oder werden mit Diskriminierung konfrontiert (z.B. Kotowska et al. 2008; Kotowska

5 Die Studentenzahl ist seit Anfang der 1990er Jahre um das Fünffache gestiegen und der Anteil der Studierenden im Alter von 19 bis 24 Jahren an allen jungen Erwachsenen dieser Altersgruppe nahm von 9,8\% im akademischen Jahr 1990/91 auf knapp 40\% im Jahr 2007/08 zu (GUS 2008b).

6 Quelle: LFS, 2. Quartal; nach Kotowska et. al. (2008: 831).

7 Unter allen Neugeborenen im Jahr 2007 gebaren die Akademikerinnen im Durchschnitt ihr 1,5. Kind. Die durchschnittliche Geburtenordnung für Frauen aller Bildungsgruppen betrug zugleich 1,8 (GUS 2008a).

8 Beispielsweise variierte im Jahr 2007 die Erwerbsquote der Frauen in der Altersgruppe 25-39 je nach Schulabschluss von $40,8 \%$ bei den niedrig- bis $86 \%$ bei den hochgebildeten Polinnen (Arbeitslosenquote entsprechend: $22,8 \%$ und $5,5 \%$ ). 
1995; Mishtal 2009). Frauen in Polen sind dabei bestrebt, gemäß der vorherrschenden Polarisierung des Erwerbsmusters, in erster Linie eine Vollzeitstelle anzunehmen (Matysiak 2007; Matysiak/Steinmetz 2006). Die Teilzeitarbeit gilt eher als eine Alternative bei fehlenden Möglichkeiten einer Vollzeitbeschäftigung. Sie wird meistens von gering qualifizierten Frauen nachgegangen und ist häufig mit einem befristeten Arbeitsverhältnis verbunden (Matysiak 2007). Im Jahr 2006 waren weniger als 10\% der 25 bis 54-jährigen Frauen teilzeiterwerbstätig.

\subsection{Institutionelle Rahmenbedingungen}

Ähnlich wie in anderen ehemaligen Ostblockstaaten sind Polinnen weiterhin nahezu alleine für Kinderbetreuung und -erziehung zuständig (CBOS 2006; Matysiak 2007). Das sozialistische Regime förderte zwar eine hohe Arbeitsmarktbeteiligung der Frauen, und dies im vollen Stundenumfang, zielte aber auf keine Veränderung der traditionellen Arbeitsteilung im Haushalt ab (Fodor et al. 2002). In den Zeiten des Sozialismus ermöglichte ein umfangreiches Paket an familien- und arbeitsmarktpolitischen Maßnahmen die Rolle einer Vollzeiterwerbstätigen mit der einer Hausfrau, relativ gut zu vereinbaren und damit die direkten Kinderkosten zu reduzieren (Frejka 2008b). In der neuen Realität Polens verschärften jedoch die weiterhin bestehende Doppelbelastung und der fortschreitende Wandel der Beschäftigungsstruktur den Konflikt zwischen den familiären und beruflichen Verpflichtungen einer Frau (Kotowska 2004, 2005; Matysiak 2005). Dieser wurde dabei keinesfalls von den bereits wirksamen oder neu eingeführten familien- und arbeitsmarktpolitischen Maßnahmen gelöst.

Wie in den meisten postsozialistischen Staaten wurden in Polen nach dem Systemumbruch eingeleitete familienpolitische Reformen einerseits dem Diktat der Einsparungen, die das Staatshaushaltsdefizit verringern sollten, und andererseits dem der Bekämpfung von wachsender Armut unterworfen (Balcerzak-Paradowska et al. 2003; Frejka 2008b; Kotowska et al. 2008). Die im Sozialismus an den Erwerbsstatus gekoppelten Sozialversicherungsleistungen mit universalem Charakter wurden größtenteils durch selektive, einkommensabhängige Beihilfen ersetzt, die effektiv nur den ärmsten Familien zugutekommen. Mit der Übertragung der Verwaltung und Finanzierung der Kinderkrippen und Kindergärten aus dem Staatsbudget an die unterfinanzierten Gemeinden, ging zudem das Angebot an Kinderbetreuungseinrichtungen erheblich zurück (Balcerzak-Paradowska et al. 2003). Die Zahl der Kinderkrippen verringerte sich im Zeitraum von 1989 bis 2007 um 76\% und die der Kindergärten um 36\%. In Anbetracht des fortschreitenden Geburtenrückgangs blieb jedoch die Versorgung mit institutionellen Kinderbetreuungseinrichtungen nahezu unverändert. Zum Zeitpunkt der letzten zwei Nationalen Volkszählungen - in den Jahren 1988 und 2002 standen jeweils der Hälfte aller Kinder im Alter von drei bis sechs Jahre ein Kindergartenplatz zur Verfügung (Matysiak/Steinmetz 2006). ${ }^{9}$ Die Verlagerung von etwa 30 bis $40 \%$ der Betreuungskosten von den Gemeinden auf die Eltern (Balcerzak-Paradowska et al. 2003),

9 Die Zahl der Kinderkrippenplätze pro 100 Kinder bis zum zweiten Lebensjahr sank von 4,5 im Jahr 1988 auf 2,5 im Jahr 2002 (Matysiak/Steinmetz 2006). Kindergrippen spielten jedoch schon in den Zeiten des Sozialismus eine untergeordnete Rolle, da die Unterversorgung mit öffentlichen Kinderkrippen mit einem ausgedehnten Erziehungsurlaub kompensiert wurde (ibd.). 
trug jedoch zur Erhöhung der direkten Kinderkosten und damit zu einer, aus finanziellen Gründen, geringeren Bereitschaft der Inanspruchnahme bei.

Die gesetzlichen Regulierungen zum Erziehungsurlaub veränderten sich seit dem Systemumbruch nur unwesentlich. Er kann weiterhin im Anschluss an den Mutterschaftsurlaub einer erwerbstätigen Mutter, und seit dem Jahr 1996 auch vom Vater, drei Jahre lang - aber maximal bis zum vierten Lebensjahr des Kindes - in Anspruch genommen werden. Die neu eingeführten Bestimmungen aus den Jahren 2002 und 2003 zur besseren Vereinbarkeit von Kind und Beruf räumen den Eltern die Möglichkeiten ein, den Erziehungsurlaub durch Teilzeitarbeit zu ersetzen oder in vier Abschnitte aufzuteilen (bis zum vierten Lebensjahr des Kindes). Die Eltern dürfen auch, solange die Betreuung der Kinder dadurch nicht erschwert wird, eine Beschäftigung aufnehmen und an Weiterbildungsmaßnahmen teilnehmen. Eine empirische Untersuchung von Matysiak (2007) zeigt allerdings, dass diese Möglichkeiten, zumindest in den ersten Jahren nach der Einführung, kaum genutzt wurden. Lediglich 3\% der Frauen ersetzten den Erziehungsurlaub durch eine Teilzeitarbeit, 7\% teilten ihn in Abschnitte auf und 5\% nahmen während der Beanspruchung an Weiterbildungsmaßnahmen teil. Der Erziehungsurlaub wird dabei hauptsächlich von Frauen in Anspruch genommen (50\% der berechtigten Mütter und 2,6\% der Väter), wobei $80 \%$ der Mütter ihn in seiner vollen Länge nutzt. Die Inanspruchnahme steigt mit sinkendem Schulabschluss deutlich an - nur knapp über ein Drittel der Frauen im Erziehungsurlaub hatten ein hohes, dafür aber $60 \%$ ein niedriges Bildungsniveau. Eine wesentliche Rolle spielt offensichtlich das Anrecht auf finanzielle Leistungen, die während des Erziehungsurlaubs bei Erfüllung des Einkommenskriteriums bezogen werden können. ${ }^{10}$ Nahezu $70 \%$ der Frauen im Erziehungsurlaub erhalten eine monetäre Leistung (Matysiak 2007). Eine fehlende Berechtigung zur Beihilfe oder zu niedrige Leistungen werden hingegen von jeder dritten Frau als Verzichtsgrund auf den Erziehungsurlaub genannt. Jede fünfte Polin macht keinen Gebrauch von dieser Maßnahme aus Angst vor deren negativen Auswirkungen auf das Berufsleben und auf das Verhältnis zu dem Arbeitsgeber (ibd.).

\subsection{Forschungshypothesen}

Vor dem Hintergrund der theoretischen Überlegungen und des landesspezifischen sozioökonomischen Kontexts lässt sich in Polen ein starker Substitutionseffekt vermuten. Die weiterhin gültige traditionelle Rollenaufteilung hinsichtlich der Kinderbetreuung bedeutet bei Geburt eines Kindes einen zumindest temporären Rückzug der Frau aus dem Erwerbsleben. Obwohl die Polinnen, zumindest im Alter, in dem die meisten familiären Entscheidungen getroffen werden, besser gebildet sind als Männer, ist ihre Position auf dem Arbeitsmarkt als eher schwierig einzustufen. Dennoch oder gerade deswegen sind sie stark bemüht berufstätig zu werden. Ein höherer Schulabschluss wirkt sich positiv auf die Erwerbschancen, die Einstellungsbedingungen und das zu erwartende Einkommen der Frau aus. Mit dem steigenden Bildungsniveau nehmen jedoch auch die Opportunitätskosten einer familienbedingten Erwerbsunterbrechung zu. Die Akademikerinnen müssen im Ver-

10 Die so genannte Beihilfe aufgrund von Kinderbetreuung wird bis zu 24 Monate in einem relativ niedrigen Pauschalbetrag ausgezahlt, der jedoch wegfällt, wenn die Eltern im Erziehungsurlaub eine Arbeit aufnehmen. 
gleich zu den geringer qualifizierten Frauen größere finanzielle Einbußen hinnehmen, die nur unwesentlich von den staatlichen Familienleistungen kompensiert werden. Darüber hinaus haben die eingeschränkte Flexibilisierung des Erziehungsurlaubs sowie dessen strikte Regulierungen zu Verdienstmöglichkeiten (beim Bezug von monetären Leistungen) zur Folge, dass viele der Frauen während der dreijährigen Inanspruchnahme nahezu vollständig den Kontakt zu ihrem beruflichen Umfeld verlieren. Die Hochschulabsolventinnen müssen dabei größere Nachteile der Entwertung des Humankapitals im Laufe einer solch langen Erwerbspause für die zukünftige Berufskarriere befürchten, als Frauen anderer Bildungsgruppen. Viele der hoch gebildeten Polinnen entscheiden demnach erst gar keinen Gebrauch vom Erziehungsurlaub zu machen, wodurch der Konflikt zwischen ihren familiären Verpflichtungen und ihrem beruflichen Engagement zusätzlich verschärft wird. Hinzu kommen die erschwerte Rückkehr in das Erwerbsleben und die Diskriminierung von Müttern mit Kleinkindern, die das Einkommen und Berufschancen der Akademikerinnen langfristig beeinflussen. Gemäß der ökonomischen Theorie der Fertilität sollen die hoch gebildeten Polinnen nach Geburt des ersten Kindes, die immer noch nahezu universal gewünscht wird, bestrebt sein, die entstandenen Kosten zu reduzieren, indem sie sich gegen ein zweites Kind entscheiden. Demzufolge wird im postsozialistischen Polen eine mit zunehmendem Bildungsniveau der Frau sinkende Zweitgeburtsintensität erwartet (Substitutionseffekt-Hypothese).

Des Weiteren verlangt die sozio-ökonomische Situation der meisten Haushalte in Polen eine Erwerbsbeteiligung beider Partner. Die Partizipation der Frau auf dem Arbeitsmarkt wirkt sich wesentlich auf das Haushaltsbudget und die Erhaltung des Lebensstandards aus. Die Akademikerinnen können über größere finanzielle Ressourcen verfügen und sind somit besser in der Lage die anfallenden, nach dem Jahr 1989 deutlich angestiegenen, direkten Kinderkosten aufzubringen. Höheres Einkommen verhilft außerdem die entstandenen Zeitkosten zu reduzieren. Die Hochschulabsolventinnen können einfacher als Polinnen niedrigerer Bildungsgruppen die kostenpflichtigen Kindergärtenplätze oder auch andere außerfamiliäre Möglichkeiten der Kinderbetreuung beanspruchen. Diese Aspekte sprechen für einen starken Einkommenseffekt in Polen, der eine mit steigendem Bildungsniveau ebenfalls zunehmende Übergangsrate zum zweiten Kind erwarten lässt (Einkommenseffekt-Hypothese).

Die folgende Studie nimmt somit an, dass sowohl der Substitutions- als auch der Einkommenseffekt in Polen stark sind. Die empirischen Analysen sollen zeigen, welcher der beiden Effekte dominiert. In einem weiteren Schritt wird zusätzlich die Gültigkeit der Time-Squeeze-Hypothese überprüft. Die hoch gebildeten Polinnen bekommen ihr erstes Kind später als Frauen anderer Bildungsgruppen. Längere Ausbildungsphase und eine Erwerbsstrategie, die auf Absicherung der Position der Frauen auf dem Arbeitsmarkt vor dem Eintritt in die Mutterschaft ausgerichtet ist, tragen unmittelbar zum Aufschub der Familiengründung bei. Folglich verkürzt sich der Zeitraum in dem angesichts der biologischen Grenze ein weiterer Kinderwunsch realisiert werden kann. Betrachtet man zusätzlich die gültigen Altersnormen für familiäre Entscheidungen - die Polinnen bekommen ihre Kinder immer noch sehr jung - sowie die negativen Folgen einer langen Erwerbsunterbrechung und die Schwierigkeiten der erwerbstätigen Mütter auf dem Arbeitsmarkt, dann ist eine Minimierung des Geburtenabstands unter den Akademikerinnen durchaus denkbar. Solch ein „Time-Squeeze“ hat dabei Implikationen für ereignisanalytische Mo- 
delle, in denen die abhängige Variable die Rate ist. Diese kann sich erhöhen, wenn sich der Geburtsabstand verkürzt, obwohl die endgültige Wahrscheinlichkeit, ein zweites Kind zu bekommen, unverändert bleibt.

\section{Empirische Analyse}

\subsection{Datensatz und Variablen}

Die Analyse des Einflusses der Bildung der Frau auf den Übergang zum zweiten Kind in Polen beruht auf dem Employment, Family and Education Survey (EFES). Der EFES wurde im Rahmen des Projektes „Cultural and structural conditions of female labour force participation in Poland" vom Institut für Statistik und Demographie (Warsaw School of Economics) konzipiert und im Jahr 2006 durchgeführt. Er stellt eine retrospektive Umfrage dar, die eine vollständige, monatsgenaue Rekonstruierung der Bildungs-, Erwerbs-, Partnerschafts-, Fertilitäts- und Migrationsbiographie der Befragten ab dem 15. Lebensjahr ermöglicht. Interviewt wurden dabei 3005 Frauen, die zwischen den Jahren 1966 und 1981 geboren wurden. Die Befragten gingen aus einer repräsentativen Stichprobe hervor.

Die folgende Studie konzentriert sich auf den Einfluss der Bildung auf das Zweitgeburtsverhalten von Frauen, die ihre bisherige Fertilitätskarriere nahezu vollständig im postsozialistischen Polen durchlebten. Die Analyse beschränkt sich daher auf die Familienentscheidungen von Frauen der Geburtskohorten 1971-1981. ${ }^{11}$ Diese waren zum Zeitpunkt des Regimewechsels zwischen dem 8. und dem 18. Lebensjahr und bei der Befragung im Jahr 2006 im Alter von 25 bis 35 Jahren. Die Stichprobe umfasst dabei nur Befragte, die ihr erstes Kind unter den veränderten sozio-ökonomischen Rahmenbedingungen, d.h. nach dem Jahr 1989, bekamen. Für das erste Kind wird das Geburtsdatum und für das zweite der Schwangerschaftsbeginn analysiert. Die Untersuchung beschränkt sich nur auf die leiblichen Kinder. Frauen, die eine Zwillingsgeburt (als erste Geburt) erfuhren, wurden jedoch aus der Stichprobe ausgeschlossen. Die derartig abgegrenzte Stichprobe ermöglicht eine Analyse der Familienbiographien von 1520 Polinnen.

Als abhängige Variable gilt die Übergangsrate zum zweiten Kind. Unter der Annahme, dass eine Schwangerschaft in den ersten Wochen nach der Entbindung eher unwahrscheinlich ist, fängt die Prozesszeit drei Monate nach der Geburt des ersten Kindes an. Als Ereignis gilt der Beginn einer zweiten Schwangerschaft mit dem die Entscheidung für eine Familienerweiterung untersucht werden soll (Št' astná 2009). ${ }^{12}$ Die Fälle, die bis zum Zeitpunkt der Befragung kein Ereignis erfuhren, wurden zensiert (Rechtszensierung). Dies gilt auch für die wenigen Frauen, die länger als zehn Jahre dem Risiko ausgesetzt

11 Zusätzlich wurde ein Versuch unternommen, die möglichen Lebenspartner der befragten Frauen zu interviewen. Da jedoch unter den 2223 Lebensgefährten lediglich 44\% bereit waren, an der Umfrage teilzunehmen, gelten die Angaben zum Partner als eher ungeeignet für wissenschaftliche Analysen.

12 In der Analyse wird angenommen, dass eine Schwangerschaft genau neun Monate dauert. Dementsprechend werden vom Geburtsdatum des zweiten Kindes neun Monate subtrahiert. Die fehlenden Angaben zum Geburtsmonat wurden mithilfe einer Zufallsvariable ergänzt. 
waren, aber kein zweites Kind bekamen (12 Fälle). Das Ereignis ist bei 771 von 1520 beobachteten Frauen eingetreten.

In dieser Studie stellt das Bildungsniveau der Frau die zentrale Erklärungsvariable des Zweitgeburtsverhaltens der Polinnen dar. Mithilfe dieser Kovariate soll das Zusammenspiel zwischen dem Einkommens- und dem Substitutionseffekt sowie die Gültigkeit der Time-Squeeze-Hypothese untersucht werden. Für die empirischen Modelle wurde der Schulabschluss der Frau als zeitabhängige Variable mit Ausprägungen: in Ausbildung und jeweils niedrige, mittlere und hohe Bildung generiert. $\mathrm{Zu}$ der niedrigen Bildungsgruppe gehören Abgängerinnen einer Grund- oder Berufsgrundschule ${ }^{13}$. Die Kategorie mittlere Bildung erfasst Frauen, die ein Allgemeinbildendes Lyzeum, eine technische bzw. Berufsbildende Mittelschule und/oder eine Postabiturschule abgeschlossen haben. Als hoch gebildet gelten Frauen mit einem universitären Abschluss. In den empirischen Modellen wird ebenfalls für die demographischen Merkmale wie Kohortenzugehörigkeit und das Alter der Frau bei der Geburt des ersten Kindes kontrolliert. Außerdem wird der Einfluss der Wohnortgröße der Frau im Alter von 15 Jahren mitberücksichtigt. Diese Variable ist relevant, da die Fertilität in den ländlichen Gebieten Polens traditionell etwas höher als in den Städten ist (GUS 2008a). Schließlich wird der Effekt der sozialen Herkunft über die Bildung der Mutter und des Vaters der befragten Frau untersucht. Alle Kovariaten sind über die Zeit konstant. Die Komposition der Stichprobe veranschaulichen Tabellen 1(a, b).

Tabelle 1(a): Deskription der Stichprobe. Verteilung der Personenmonate, Frauenzahl und des Ereignisses auf die verschiedenen Ausprägungen der erklärenden Variablen

\begin{tabular}{|c|c|c|c|c|c|}
\hline \multirow[t]{2}{*}{ Erklärende Variable } & \multicolumn{2}{|c|}{ Personenmonate } & \multicolumn{2}{|c|}{ Frauenzahl } & \multirow[t]{2}{*}{ Zweites Kind } \\
\hline & absolut & $\%$ & absolut & $\%$ & \\
\hline \multicolumn{6}{|l|}{ Kohorte } \\
\hline 1971-1975 & 41329 & 57.46 & 767 & 50.46 & 464 \\
\hline $1976-1981$ & 30596 & 42.54 & 753 & 49.54 & 307 \\
\hline \multicolumn{6}{|l|}{ Bildung der befragten Frau } \\
\hline in Ausbildung & 6388 & 8.88 & - & - & 41 \\
\hline Grund- und Berufsgrundschule & 26487 & 36.83 & - & - & 379 \\
\hline Mittlere Bildung $\left(^{*}\right)$ & 28646 & 39.83 & - & - & 284 \\
\hline Hochschulabschluss & 10404 & 14.47 & - & - & 67 \\
\hline \multicolumn{6}{|c|}{ Alter der Frau bei Geburt des ersten Kindes } \\
\hline über 15 bis 19 & 6253 & 8.69 & 118 & 7.76 & 87 \\
\hline über 19 bis 22 & 27571 & 38.33 & 488 & 32.11 & 339 \\
\hline über 22 bis 26 & 27557 & 38.31 & 593 & 39.01 & 275 \\
\hline über 26 bis 35 & 10544 & 14.66 & 321 & 21.12 & 70 \\
\hline \multicolumn{6}{|c|}{ Wohnortgröße der Frau im Alter von 15 Jahren } \\
\hline$>500.000$ Einw. & 5351 & 7.44 & 118 & 7.76 & 36 \\
\hline 100.000-500.000 Einw. & 11245 & 15.63 & 205 & 13.49 & 84 \\
\hline$<100.000$ Einw. & 26314 & 36.59 & 521 & $34-28$ & 254 \\
\hline Dorf & 27181 & 37.79 & 629 & 41.38 & 371 \\
\hline Missing & 1834 & 2.55 & 49 & 3.22 & 1.26 \\
\hline
\end{tabular}

13 Die erstellten Bildungskategorien beziehen sich auf das aus den Zeiten des Sozialismus stammende Schulsystem Polens. Dies wurde im Jahr 1999 grundlegend reformiert. 


\begin{tabular}{|c|c|c|c|c|c|}
\hline \multirow[t]{2}{*}{ Erklärende Variable } & \multicolumn{2}{|c|}{ Personenmonate } & \multicolumn{2}{|c|}{ Frauenzahl } & \multirow[t]{2}{*}{ Zweites Kind } \\
\hline & absolut & $\%$ & absolut & $\%$ & \\
\hline \multicolumn{6}{|l|}{ Bildung der Mutter } \\
\hline niedrige Bildung & 19856 & 27.61 & 443 & 29.14 & 281 \\
\hline Berufsgrundschule & 26083 & 36.26 & 536 & 35.26 & 284 \\
\hline mittlere Bildung & 19635 & 27.3 & 413 & 27.17 & 168 \\
\hline hohe Bildung & 4384 & 6.1 & 97 & 6.38 & 27 \\
\hline Missing & 1967 & 2.73 & 31 & 2.04 & 11 \\
\hline \multicolumn{6}{|l|}{ Bildung des Vaters } \\
\hline niedrige Bildung & 17155 & 23.85 & 397 & 26.12 & 246 \\
\hline Berufsgrundschule & 34182 & 47.52 & 704 & 46.32 & 361 \\
\hline mittlere Bildung & 14357 & 19.96 & 297 & 19.54 & 124 \\
\hline hohe Bildung & 4262 & 5.93 & 90 & 5.92 & 27 \\
\hline Missing & 1969 & 2.74 & 32 & 2.11 & 13 \\
\hline \multicolumn{6}{|l|}{ Fallzahlen } \\
\hline Frauen & & & & & 1520 \\
\hline Personenmonate & & & & & 71925 \\
\hline Ereignis (zweite Schwangerschaft) & & & & & 771 \\
\hline
\end{tabular}

Tabelle 1(b): Deskription der Stichprobe. Frauenzahl nach Bildung der befragten Frau. Zeitveränderliche Bildungsvariable zu Episodenbeginn

\begin{tabular}{|c|c|c|c|c|c|c|c|c|c|c|}
\hline \multirow[b]{3}{*}{ Bildung der befragten Frau } & \multicolumn{10}{|c|}{ Alter des ersten Kindes } \\
\hline & \multicolumn{2}{|c|}{$\begin{array}{l}0 \text { bis unter } \\
1 \mathrm{Jahr}\end{array}$} & \multicolumn{2}{|c|}{$\begin{array}{l}1 \text { bis unter } \\
2 \text { Jahre }\end{array}$} & \multicolumn{2}{|c|}{$\begin{array}{l}2 \text { bis unter } \\
4 \text { Jahre }\end{array}$} & \multicolumn{2}{|c|}{$\begin{array}{c}4 \text { bis unter } \\
6 \text { Jahre }\end{array}$} & \multicolumn{2}{|c|}{$\begin{array}{c}6 \text { bis unter } \\
10 \text { Jahre }\end{array}$} \\
\hline & $\begin{array}{l}\text { abso- } \\
\text { lut }\end{array}$ & in $(\%)$ & $\begin{array}{l}\text { abso- } \\
\text { lut }\end{array}$ & in $(\%)$ & $\begin{array}{l}\text { abso- } \\
\text { lut }\end{array}$ & in $(\%)$ & $\begin{array}{c}\text { abso- } \\
\text { lut }\end{array}$ & in $(\%)$ & $\begin{array}{l}\text { abso- } \\
\text { lut }\end{array}$ & in $(\%)$ \\
\hline in Ausbildng & 221 & 14.54 & 141 & 11.08 & 92 & 9.06 & 41 & 6.69 & 23 & 6.34 \\
\hline $\begin{array}{l}\text { Grund- und Berufsgrund- } \\
\text { schule }\end{array}$ & 563 & 37.04 & 471 & 37.03 & 374 & 36.81 & 220 & 35.89 & 129 & 35.54 \\
\hline Mittlere Bildung $\left(^{*}\right)$ & 529 & 34.80 & 481 & 37.81 & 411 & 40.45 & 261 & 42.58 & 155 & 42.70 \\
\hline Hochschulabschluss & 207 & 13.62 & 179 & 14.07 & 139 & 13.68 & 91 & 14.85 & 56 & 15.43 \\
\hline $\begin{array}{l}\text { Frauenzahl zu } \\
\text { Episodenbeginn }\end{array}$ & 1,520 & & 1,272 & & 1.016 & & 613 & & 363 & \\
\hline
\end{tabular}

(*) Mittlere Bildung: Abschluss eines Allgemeinbildenden Lyzeums, einer technischen bzw. berufsbildenden Mittelschule und/oder einer Postabiturschule.

Quelle: Employment, Family and Education Survey 2006; eigene Berechnungen.

\subsection{Methode}

Für die empirische Untersuchung wird auf die Methoden und Techniken der Ereignisdatenanalyse zurückgegriffen. Im ersten Schritt wird der Übergang zum zweiten Kind anhand von Survivalkurven dargestellt, die mittels Kaplan-Meier-Verfahren geschätzt wurden. Im zweiten Schritt wird anhand eines proportionalen Hazard-Modells der gleichzeitige Einfluss mehrerer Kovariaten auf den Übergang zum zweiten Kind ermittelt. Das Modell lässt sich mathematisch folgendermaßen darstellen: 


$$
h(t \mid X)=h_{0}(t) \exp \left(\sum_{i=1}^{p} \beta_{i} X_{i}\right) \text { mit } X=\left(X_{1}, X_{2}, \ldots, X_{p}\right)
$$

wobei $h_{0}(t)$ die Baseline-Hazardfunktion beschreibt. $\beta_{i}$ steht für die unbekannten und zu schätzenden Regressionsparameter und $X_{i}$ für die bekannten Kovariaten. In dieser Studie wird die Übergangsrate zum zweiten Kind anhand eines so genannten Piecewise-Constant-Exponential-Modells geschätzt. Dem Modell liegt ein Exponentialmodell zugrunde, jedoch mit dem Unterschied, dass die Baseline-Hazard in mehrere vorgegebene Intervalle unterteilt wird. Entsprechend handelt sich um eine Baseline-Hazard, die zwischen den Segmenten variieren kann, innerhalb der einzelnen Intervalle jedoch immer konstant verläuft. Für die Untersuchung der Zweitgeburtsintensität in Polen wird die Baseline-Hazard über das Alter des ersten Kindes bei der zweiten Schwangerschaft definiert. Das Risiko der zweiten Empfängnis soll per Annahme für die folgenden Jahressegmente konstant bleiben: unter einem Jahr, ein Jahr bis unter zwei Jahre, zwei Jahre bis unter vier Jahre, vier bis unter sechs Jahre und sechs Jahre bis unter zehn Jahre. Zur Schätzung der Koeffizientenwerte wird die Maximum-Likelihood-Methode herangezogen. In diesem Schätzverfahren werden die Werte für die unbekannten Parameter ermittelt, bei denen das beobachtete Stichprobenergebnis am wahrscheinlichsten ist. Das ereignisanalytische Modell wird mit Hilfe der statistischen Software STATA geschätzt.

\subsection{Ergebnisse}

\section{Deskriptive Darstellung}

Die Abbildung 1 zeigt den Verlauf einer mittels Kaplan-Meier Methode geschätzten Survivalfunktion für den Übergang zum zweiten Kind in Polen. Der Median des Geburtenabstandes beträgt 58 Monate, d.h. die Hälfte der Frauen wurde 4,8 Jahre nach der ersten Mutterschaft mit dem zweiten Kind schwanger. Das Ereignis ist bei einem Drittel der Mütter (32\%) zehn Jahre nach der Geburt des ersten Kindes immer noch nicht eingetreten. Die in der Abbildung 2 dargestellten Survivalverläufe bringen erhebliche Unterschiede im Zweitgeburtsverhalten hinsichtlich des Alters der Frau bei Geburt des ersten Kindes zum Vorschein. Je jünger die Polin bei der Familiengründung ist, umso niedriger fällt der Median des Geburtenabstandes aus. Er liegt bei 44 Monaten (3,7 Jahre) bei den Frauen, die ihr erstes Kind im Alter von 15 bis 19 Jahren bekommen und dementgegen bei 89 Monate (7,4 Jahre) bei denjenigen, die ihre erste Mutterschaft erst nach dem 26. Lebensjahr realisieren. Interessanterweise wurde zehn Jahre nach der ersten Mutterschaft nur ein Viertel aller Frauen, die im Alter von über 15 bis 19 und über 19 bis 22 ihr erstes Kind bekamen, noch nicht erneut schwanger. Von den Polinnen, die im Alter über 22 bis 26 Mutter wurden, erfuhren im selben Zeitraum etwa 40\% noch kein Ereignis. Die Unterschiede beim Übergang zur zweiten Schwangerschaft zwischen den Frauen verschiedener Altersgruppen bei Geburt des ersten Kindes sind laut des Log-rank-Tests hoch signifikant. 
Abbildung 1: Kaplan-Meier Schätzer für den Übergang zum zweiten Kind in Polen

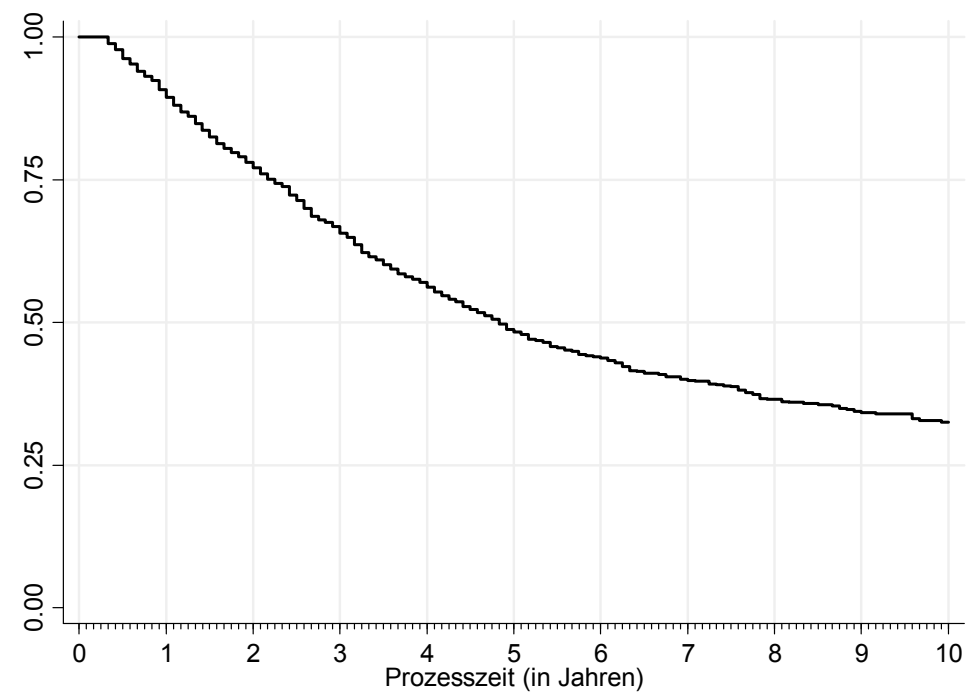

Quelle: Employment, Family and Education Survey 2006; eigene Berechnungen.

Abbildung 2: $\quad$ Kaplan-Meier Schätzer für den Übergang zum zweiten Kind in Polen nach Alter bei Geburt des ersten Kindes

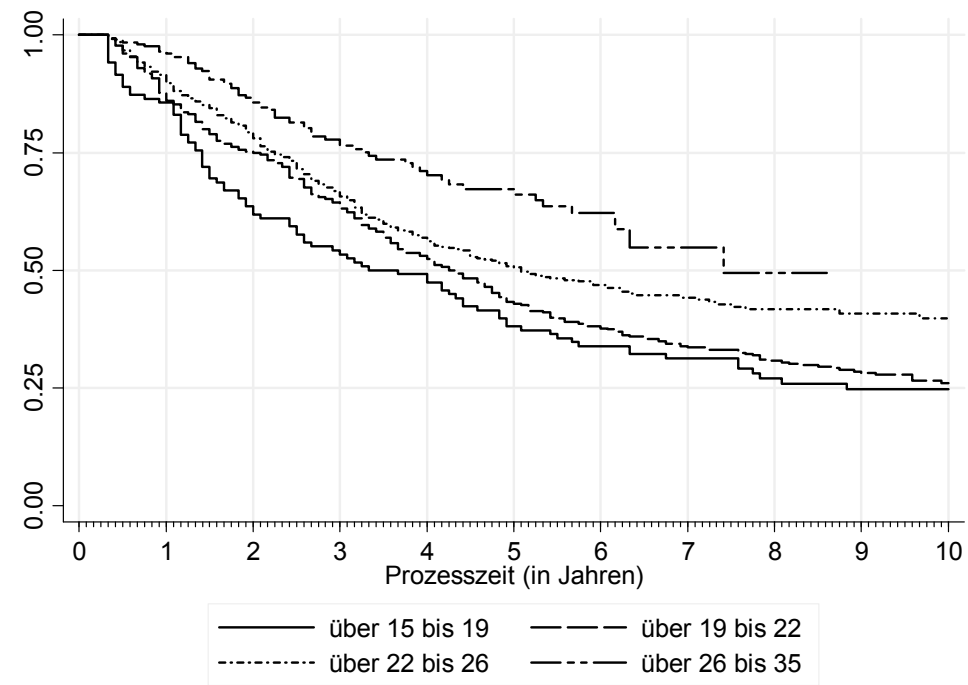

Quelle: Employment, Family and Education Survey 2006; eigene Berechnungen. 
Multivariate Analyse

In bisherigen Analysen wurden die Effekte einzelner Kovariaten auf das Zweitgeburtenverhalten in Polen getrennt voneinander betrachtet. In einem weiteren Schritt soll deren gleichzeitiger Einfluss mit besonderem Augenmerk auf den Bildungseffekt untersucht werden. Dazu werden insgesamt vier Piecewise-Constant-Exponential-Modelle geschätzt, deren Ergebnisse die Tabelle 2 dargestellt. Ausgehend vom Modell 1, das lediglich für die Kohorte und die Bildung der befragten Frau kontrolliert, werden schrittweise weitere erklärende Variable eingefügt.

Das erste Modell zeigt, dass die Übergangsrate zur zweiten Schwangerschaft mit dem Alter des ersten Kindes, wenn für Kohortenzugehörigkeit und Bildungsniveau der Frau kontrolliert wird, eine Glockenform annimmt. Die Zweitgeburtsintensität ist am höchsten, wenn das erste Kind im Alter von zwei bis vier Jahren ist. Die Polinnen jüngerer Jahrgänge weisen eine signifikant geringere Übergangsrate zum zweiten Kind auf als Frauen älterer Geburtskohorten. Mit dem steigenden Bildungsniveau der Frau nimmt das Risiko einer zweiten Schwangerschaft signifikant ab. Bezugnehmend auf die Referenzkategorie (niedrige Bildung) haben die Akademikerinnen eine um 56\% und die mittel gebildeten Frauen eine um 31\% geringere Zweitgeburtsintensität. Das Modell zeigt auch, dass Frauen in der Ausbildungsphase ebenfalls ein signifikant geringeres Risiko einer zweiten Schwangerschaft haben als die niedrig gebildeten Polinnen. Im zweiten Modell wird zusätzlich für das Alter der Frau bei Geburt des ersten Kindes kontrolliert. Hier wird der negative Einfluss des steigenden Alters bei Familiengründung auf das Zweitgeburtsverhalten sichtbar. Polinnen, die das erste Kind im Alter von 22 bis 26 Jahren bekommen, zeigen eine um $17 \%$ signifikant geringere Übergangsrate zum zweiten Kind als Frauen der Referenzkategorie (Geburt des ersten Kindes im Alter von 19 bis 22 Jahre). Ein noch stärkerer Effekt lässt sich für Frauen feststellen, die ihr erstes Kind erst nach dem 26. Lebensjahr realisieren. Solch ein später Eintritt in die Mutterschaft senkt die Zweitgeburtsintensität sogar um $42 \%$ im Vergleich zur Referenzkategorie. Durch die Aufnahme des Alters der Frau bei Geburt des ersten Kindes in das Modell fällt der Bildungseffekt wesentlich schwächer aus, der negative Bildungsgradient bleibt jedoch weiterhin bestehen. In das dritte Modell wird der Einfluss der Wohnortgröße der Frau im Alter von 15 Jahren eingefügt. Die Übergangsrate zur zweiten Schwangerschaft sinkt signifikant mit zunehmender Größe des Ortes, in dem die Frau aufwuchs. Die Berücksichtigung dieser Variable im Modell verringert ebenfalls den negativen Bildungseffekt und den des Verweilens in der Ausbildungsphase. Schließlich wird die Bildung der Mutter und die des Vaters der befragten Frau in das vierte Modell miteinbezogen. Diese Elterncharakteristika üben keinen signifikanten Einfluss auf die Übergangsrate zum zweiten Kind aus, reduzieren jedoch weiter den negativen Effekt der steigenden Bildung der Frau. Demnach weisen die Akademikerinnen eine um 31\% und die mittel gebildeten Frauen eine um 16\% niedrigere Zweitgeburtsintensität auf als Frauen der Referenzkategorie. Eine ähnliche Abschwächung lässt sich auch für den negativen Effekt des Verweilens in der Ausbildungsphase feststellen. Polinnen, die im Bildungssystem verweilen, weisen eine um $47 \%$ geringere Zweitgeburtsrate als die niedrig gebildeten Frauen auf. 
Tabelle 2: Relative Risiken des Übergangs zum zweiten Kind in Polen. Ergebnisse eines Piecewise-Constant-Exponential Modells

\begin{tabular}{|c|c|c|c|c|}
\hline Erklärende Variable & Modell1 & Modell 2 & Modell 3 & Modell4 \\
\hline \multicolumn{5}{|l|}{ Alter des ersten Kindes } \\
\hline 0 bis unter 1 Jahr & 1 & 1 & 1 & 1 \\
\hline 1 bis unter 2 Jahre & $1.36^{* * *}$ & $1.34^{* * *}$ & $1.36^{* * *}$ & $1.36^{* * *}$ \\
\hline 2 bis unter 4 Jahre & $1.44^{* * *}$ & $1.39^{* * *}$ & $1.42^{\star * *}$ & $1.42^{* * *}$ \\
\hline 4 bis unter 6 Jahre & 1.17 & 1.11 & 1.14 & 1.14 \\
\hline 6 bis unter 10 Jahre & $0.70^{\star *}$ & $0.64^{* *}$ & $0.66^{\star * *}$ & $0.66^{\star \star \star}$ \\
\hline \multicolumn{5}{|l|}{ Kohorte } \\
\hline 1971-1975 & 1 & 1 & 1 & 1 \\
\hline $1976-1981$ & $0.86^{\star *}$ & $0.81^{* * *}$ & $0.81^{* * *}$ & $0.80^{* * *}$ \\
\hline \multicolumn{5}{|l|}{ Bildung der befragten Frau } \\
\hline in Ausbildung & $0.45^{* * *}$ & $0.46^{\star * *}$ & $0.5^{* * *}$ & $0.53^{\star \star \star}$ \\
\hline $\begin{array}{l}\text { Grund- und Berufsgrund- } \\
\text { schule }\end{array}$ & 1 & 1 & 1 & 1 \\
\hline mittlere Bildung $\left(^{*}\right)$ & $0.69^{* * *}$ & $0.74^{* * *}$ & $0.80^{* * *}$ & $0.84^{* *}$ \\
\hline Hochschulabschluss & $0.44^{* * *}$ & $0.56^{* * *}$ & $0.64^{* * *}$ & $0.69^{* *}$ \\
\hline \multicolumn{5}{|l|}{ Alter der Frau bei Geburt des } \\
\hline \multicolumn{5}{|l|}{ ersten Kindes } \\
\hline über 15 bis 19 & & 1.13 & 1.16 & 1.18 \\
\hline über 19 bis 22 & & 1 & 1 & 1 \\
\hline über 22 bis 26 & & $0.83^{* * *}$ & $0.82^{\star * *}$ & $0.81^{* * *}$ \\
\hline über 26 bis 35 & & $0.58^{* * *}$ & $0.59^{* * *}$ & $0.58^{* * *}$ \\
\hline \multicolumn{5}{|l|}{$\begin{array}{l}\text { Wohnortgröße der Frau im Al- } \\
\text { ter von } 15 \text { Jahren }\end{array}$} \\
\hline > 500.000 Einw. & & & $0.58^{\star * *}$ & $0.63^{* * *}$ \\
\hline 100.000-500.000 Einw. & & & $0.62^{\star * *}$ & $0.65^{\star * *}$ \\
\hline$<100.000$ Einw. & & & $0.78^{* * *}$ & $0.82^{* *}$ \\
\hline Dorf & & & 1 & 1 \\
\hline Missing & & & 1.12 & 1.12 \\
\hline \multicolumn{5}{|l|}{ Bildung der Mutter } \\
\hline niedrige Bildung & & & & 1.11 \\
\hline Berufsgrundschule & & & & 1 \\
\hline mittlere Bildung & & & & 1.03 \\
\hline hohe Bildung & & & & 0.92 \\
\hline Missing & & & & 0.52 \\
\hline \multicolumn{5}{|l|}{ Bildung des Vaters } \\
\hline niedrige Bildung & & & & 1.1 \\
\hline Berufsgrundschule & & & & 1 \\
\hline mittlere Bildung & & & & 1.03 \\
\hline hohe Bildung & & & & 0.93 \\
\hline Missing & & & & 1.16 \\
\hline Log likelihood (Baseline) & -1759.664 & -1758.664 & -1757.664 & -1757.664 \\
\hline Log likelihood (Endwert) & -1707.063 & -1696.737 & -1683.427 & -1678.775 \\
\hline
\end{tabular}

Signifikanzniveau: $* * * p \leq 0,01 ; * * p \leq 0,05 ; * p \leq 0,1$.

(*) Mittlere Bildung: Abschluss eines Allgemeinbildenden Lyzeums, einer technischen bzw. berufsbildenden Mittelschule und/oder einer Postabiturschule.

Quelle: Employment, Family and Education Survey 2006; eigene Berechnungen. 
Die Befunde der multivariaten Analysen zeigen einen signifikant negativen Effekt der steigenden Bildung der Polinnen auf ihr Zweitgeburtsverhalten. Bezugnehmend auf die aufgestellten Hypothesen fällt damit der Substitutionseffekt stärker als der Einkommenseffekt aus. Die Akademikerinnen sind folglich mit hohen Opportunitätskosten der Kindererziehung konfrontiert, die nicht ausreichend von den geltenden familien- und arbeitsmarktpolitischen Regulierungen reduziert werden. Im nächsten Schritt soll die Gültigkeit der Time-Squeeze-Hypothese für Polen untersucht werden. Solch eine Minimierung des Geburtenabstandes unter den hoch gebildeten Frauen wird aufgrund ihres höheren Alters bei der ersten Mutterschaft und dadurch auch verkürzter reproduktiver Phase angenommen. Die Abbildung 3 veranschaulicht, dass das Alter der Polinnen bei Geburt des ersten Kindes mit zunehmendem Schulabschluss tatsächlich ansteigt und unter den Akademikerinnen am höchsten ist. Weiterhin könnten auch die in Polen geltenden Altersnormen und die negativen Folgen einer langen familienbedingten Pause auf die zukünftige Berufskarriere die Hochschulabsolventinnen dazu verleiten, ihre gewünschte Kinderzahl in einem engen Zeitraum direkt nacheinander zu realisieren.

Abbildung 3: Durchschnittliches Alter bei Geburt des ersten Kindes nach Bildung der befragten Frau

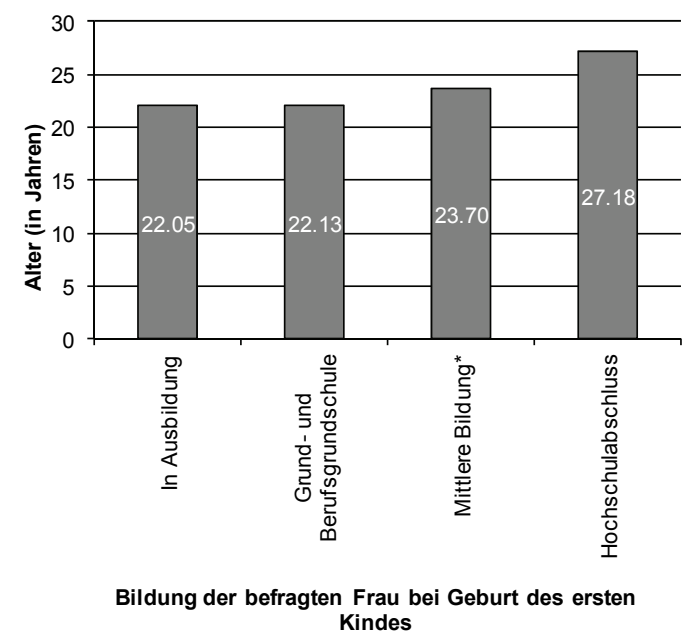

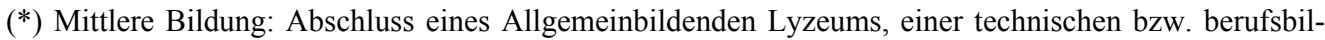
denden Mittelschule und/oder einer Postabiturschule.

Quelle: Employment, Family and Education Survey 2006; eigene Berechnungen.

Die Abbildung 4 stellt den Interaktionseffekt zwischen dem Alter des ersten Kindes (Baseline-Hazard) und dem Schulabschluss der befragten Frau dar. Im Falle eines TimeSqueeze sollte die Übergangsrate zum zweiten Kind bei den Akademikerinnen ihren Höchstwert vor dem Maximum der niedrig gebildeten Frauen aufweisen. In Polen weisen die niedrig gebildeten Frauen über die ganze Prozesszeit die höchste Übergangsrate zum zweiten Kind auf. Die Zweitgeburtsintensität ist ein bis zwei Jahre nach der Geburt des ersten Kindes besonders hoch und sinkt erst rapide ab, wenn das erste Kind sechs Jahre alt ist. Die Hochschulabsolventinnen haben durchgehend das niedrigste Risiko einer zweiten 
Schwangerschaft, mit dem Maximum zwei bis vier Jahre nach der ersten Mutterschaft. Die beschriebene Interaktion lässt somit nicht den Schluss zu, dass in Polen hoch qualifizierte Frauen einen geringen Geburtsabstand zwischen ersten und zweiten Kind wählen, so wie dies für andere Länder, etwa Frankreich (Köppen 2006), gezeigt wurde.

Abbildung 4: Interaktion zwischen dem Alter des ersten Kindes und der Bildung der befragten Frau

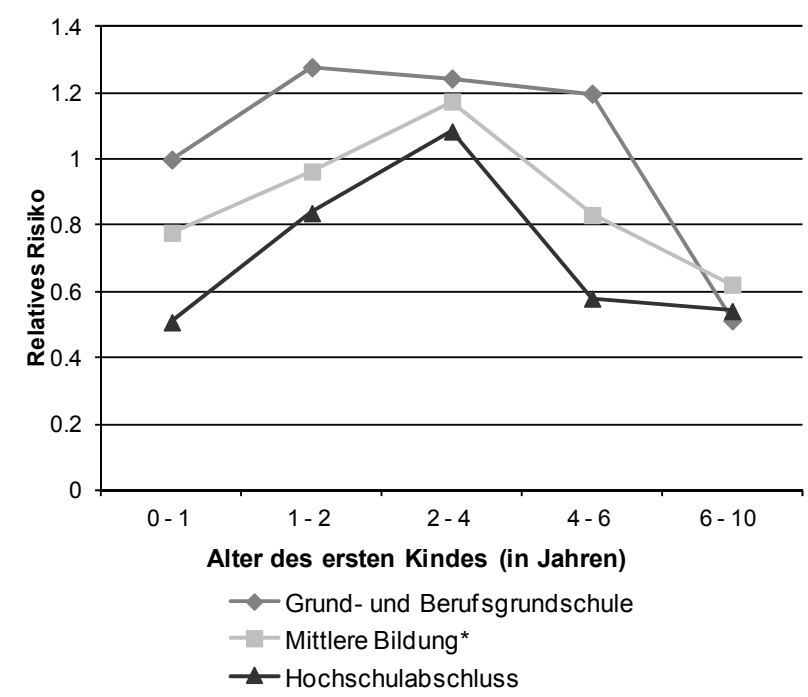

Kontrollvariablen: Kohortenzugehörigkeit, Bildung der Mutter, Bildung des Vaters, Alter der Frau bei Geburt des ersten Kindes, Wohnortgröße der Frau im Alter von 15 Jahren. Für alle Gruppen gilt die Referenzkategorie: Alter des ersten Kindes 0 bis unter 1 und Grund- und Berufsgrundschule.

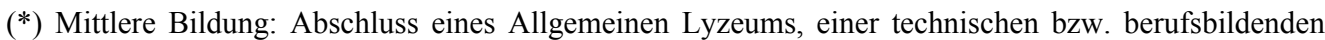
Mittelschule und/oder einer Postabiturschule.

Quelle: Employment, Family and Education Survey 2006; eigene Berechnungen.

\section{Schlussfolgerungen und Ausblick}

Nach den Ergebnissen dieser Studie lässt sich Polen international unter den meisten osteuropäischen Ländern platzieren, in denen - mit Ausnahme von Estland und der Tschechischen Republik - die Zweitgeburtsintensität mit dem zunehmenden Schulabschluss der Frau signifikant sinkt. Der negative Bildungseffekt fällt in Polen schwächer aus, bleibt jedoch weiterhin bestehen, wenn für das Alter der Frau bei der Geburt des ersten Kindes, Wohnortgröße der Frau im Alter von 15 Jahren und Bildung der Eltern der Befragten kontrolliert wird. Entsprechend ist die Zweitgeburtsintensität der Akademikerinnen um 31\% und die der mittel gebildeten Frauen um 16\% geringer als die der niedrig gebildeten Polinnen (Modell 4). Diese Befunde scheinen für eine Dominanz des Substitutions- über den Einkommenseffekt in Polen zu sprechen. Familienpolitische Maßnahmen und die Ar- 
beitsmarktregulierungen können nur eingeschränkt die hohen Opportunitätskosten der Kindererziehung reduzieren. Ein starker Substitutionseffekt impliziert beträchtliche Schwierigkeiten in der Vereinbarkeit von Kind und Beruf, mit denen die Hochschulabsolventinnen offensichtlich stärker konfrontiert sind als Frauen anderer Bildungsgruppen. Die Überprüfung der Time-Squeeze-Hypothese in Polen gab keine Hinweise darauf, dass die Hochschulabsolventinnen aufgrund ihres höheren Alters bei der ersten Mutterschaft und der daraus resultierenden verkürzten reproduktiven Phase den Geburtenabstand minimieren, um die gewünschte Kinderzahl zu realisieren. Dies kann mit einem relativ jungen Alter der Akademikerinnen bei Geburt des ersten Kindes begründet werden; hoch gebildete Frauen haben noch genug Zeit um ihre Familie zu erweitern. ${ }^{14}$

Die empirischen Befunde aus anderen Ländern deuten auf einen starken Einfluss der Partnercharakteristika auf die Übergangsrate zum zweiten Kind hin, die einen einst nachgewiesenen Bildungseffekt entscheidend schwächen oder sogar ganz verschwinden lassen können (z.B. Gerster et al. 2007; Köppen 2006; Kreyenfeld 2002). Dieser Aspekt konnte hier aufgrund von Einschränkungen des Datensatzes nicht untersucht und muss mit anderen Daten in der weiterführenden Forschung noch berücksichtigt werden. Außerdem zeigen zahlreiche Studien, dass die Operationalisierung der Selektionseffekte (unbeobachtete Heterogenität) wesentliche Auswirkungen auf empirische Modelle und damit auch auf den geschätzten Bildungseffekt haben kann (Kreyenfeld 2002; Kravdal 2001). In dem vorliegenden Beitrag wurde, gemäß der ökonomischen Theorie, die Annahme getroffen, dass die Präferenzen aller Frauen gleich und über die Zeit konstant sind. Die Bildung der Frau gilt hier als Proxy für ihr Verdienstpotential. Die Fertilitätsentscheidungen einer Frau können jedoch neben der finanziellen Situation, auch von den individuellen Familien- und Berufsorientierungen der Frau abhängen (Hakim 2003, 2000). Dies dürfte erklären, warum manche Frauen sich gegen ein weiteres Kind entscheiden, obwohl bei ihnen, ökonomisch betrachtet, der Einkommens- den Substitutionseffekt übersteigt (Matysiak 2009b: 73). Die Analyse der Rolle der Präferenzen zusammen mit der Berücksichtigung der Partnercharakteristika könnte den negativen Bildungsgradient entscheidend beeinflussen. Die Aufgabe zukünftiger Studien ist es diese Effekte genauer zu erkunden, denn sie positionieren Polen womöglich international mit Estland und der Tschechischen Republik und damit auch mit den westeuropäischen und skandinavischen Ländern.

\section{Danksagung}

Mein ganz besonderer Dank gilt Michaela Kreyenfeld und Anna Matysiak für ihre kritischen Kommentare und zahlreichen Anregungen. Für wertvolle Hinweise möchte ich

14 Bei der Interpretation der Ergebnisse sei jedoch zu beachten, dass die betrachteten Frauen immer noch in ihrer reproduktiven Phase sind. Außerdem waren Frauen, die dem Risiko einer zweiten Schwangerschaft ausgesetzt sind, womöglich selektiv. Dies betrifft vor allem Polinnen jüngerer Jahrgänge. Wenn sie in der Stichprobe enthalten sind, bedeutet das, dass sie das erste Kind sehr früh in ihrem Leben bekamen. Sie mögen eher niedrig gebildet sein, denn die Akademikerinnen gründen ihre Familien meist in einem höheren Alter. Frauen hingegen, die ihr erstes Kind nach dem 26. Lebensjahr gebaren, sind in der Risikopopulation unterrepräsentiert. Entsprechend wird das Zweitgeburtsrisiko für höher gebildete Frauen möglicherweise unterschätzt. 
meinen Dank auch Monika Mynarska und Katja Köppen sowie den anonymen Gutachter(inne)n für ihre Verbesserungsvorschläge aussprechen.

\section{Literatur}

Aisenbrey, S., Evertsson, M. \& Grunow, D. (2009). Is there a career penalty for mothers' time out? A comparison of Germany, Sweden and the United States. Social Forces, 88, 2, S. 573-605.

Albrecht, J. W., Edin, P.-A., Sundström, M. \& Vroman, S. B. (1999). Career interruptions and subsequent earnings: A reexamination using Swedish data. Journal of Human Resources, 34, 2, S. 294-311.

Balcerzak-Paradowska, B., Chłoń-Domińczak, A., Kotowska, I. E., Olejniczuk-Merta, A., Topińska, I. \& Wóycicka, I. (2003). The gender dimensions of social security reform in Poland. In: Fultz, E., Ruck, M. \& Steinhilber, S. (Hrsg.), The gender dimensions of social security reform in Central and Eastern Europe: Case studies of the Czech Republic, Hungary and Poland. Budapest: International Labour Organization, Subregional Office for Central and Eastern Europe, S. 187-313.

Becker, G. S. (1965). A theory of the allocation of time. The Economic Journal, 75, 299, S. 493-517.

Becker, G. S. (1993). A treatise on the family. London: Harvard University Press.

Blossfeld, H.-P. (1995). The new role of women: Family formation in modern societies: Boulder, CO: Westview

Blossfeld, H.-P. \& Huinink, J. (1991). Human capital investments or norms of role transition? How women's schooling and career affect the process of family formation. The American Journal of Sociology, 97, 1, S: 143-68.

CBOS (2006). Kobiety i mężczyźni o podziale obowiazków domowych [Frauen und Männer über die Arbeitsteilung im Haushalt]. Warschau: CBOS (BS/183/2006, Forschungsnotiz, Dezember 2006).

Council of Europe (2006). Recent demographic developments in Europe. Strasbourg: Council of Europe Press.

Demographic Research (2008). Special collection 7: Childbearing trends and policies in Europe. Rostock: Max Planck Institute for Demographic Research.

Ermisch, J. F. (2003). An economic analysis of the family. Princeton, Oxford: Princeton University Press.

Fodor, E., Glass, C., Kawachi, J. \& Popescu, L. (2002). Family policies and gender in Hungary, Poland and Romania. Communist and Post-Communist Studies, 35, S. 475-490.

Frejka, T. (2008a). Overview chapter 2: Parity distribution and completed family size in Europe Incipient decline of the two-child family model. Demographic Research, 19, 4, S. 47-72.

Frejka, T. (2008b). Overview chapter 5: Determinants of family formation and childbearing during the societal transition in Central and Eastern Europe. Demographic Research, 19, 7, S. 139-170.

Frejka, T. \& Sardon, J.-P. (2007). Cohort birth order, parity progression ratio and parity distribution trends in developed countries. Demographic Research, 16, 11, S. 315-374.

Frejka, T. \& Sobotka, T. (2008). Fertility in Europe. Diverse, delayed and below replacement. Demographic Research, 19, 3, S. 15-46.

Gerster, M., Keiding, N., Knudsen, L. B. \& Strandberg-Larsen, K. (2007). Education and second birth rates in Denmark 1981-1994. Demographic Research, 17, 8, S. 181-210.

Gornick, J. C., Meyers, M. K. \& Ross, K. E. (1998). Public policies and the employment of mothers: A cross-national study. Social Science Quarterly, 79, 11, S. 35-54.

GUS (2008a): Rocznik demograficzny 2008 [Demographisches Jahrbuch 2008]. Warschau: ZWS.

GUS (2008b). Szkoty wyższe $i$ ich finanse w 2007 r. [Hochschulen und ihre Finanzen im Jahre 2007]. Warschau: ZWS.

Hakim, C. (2000). Work-lifestyle choices in the 21st century. Preference theory. Oxford: Oxford University Press.

Hakim, C. (2003). A new approach to explaining fertility patterns: Preference theory. Population and Development Review, 29, S. 349-374.

Hoem, B. \& Hoem, J. M. (1989). The impact of women's employment on second and third births in modern Sweden. Population Studies, 43, 1, S.47-67. 
Kantorová, V. (2004). Education and entry into motherhood: The Czech Republic during state socialism and the transition period (1970-1997). Demographic Research, Special collection 3, 10, S. 243-274.

Klein, T. \& Lauterbach. W. (1994). Bildungseinflüsse auf Heirat, die Geburt des ersten Kindes und die Erwerbsunterbrechung von Frauen: eine empirische Analyse familienökonomischer Erklärungsmuster. Kölner Zeitschrift für Soziologie und Sozialpsychologie, 46, 2, S. 278-298.

Klesment, M. \& Puur, A. (2010). Effects of education on second births before and after societal transition: Evidence from the Estonian GGS. Demographic Research.22, 28, S. 891-932

Kohler, H.-P., Billari, F. C. \& Ortega, J. A. (2002). The emergence of lowest-low fertility in Europe during the 1990s. Population and Development Review, 28, 4, S. 641-680.

Köppen, K. (2006): Second births in western Germany and France. Demographic Research, 14. 14, S. 295-330.

Kotowska, I., Józwiak, J., Matysiak, \& Baranowska, A. (2008). Poland: Fertility decline as a response to profound societal and labour market changes? Demographic Research, 19, 22, S. 795-854

Kotowska, I. E. (1995). Discrimination against women in the labor market in Poland during the transition to a market economy. Social Politics, 2, 1, S. 76-90.

Kotowska, I. E. (1999). Przemiany demograficzne w Polsce $w$ latach 90. $w$ świetle koncepcji drugiego przejścia demograficznego [Demographische Veränderungen in Polen in den 90er Jahren im Lichte des Konzepts des zweiten demographischen Übergangs]. Warschau: Szkola Główna Handlowa.

Kotowska, I. E. (2004). Fertility and nuptiality in the CEE countries in the context of weakening families and a weakening state. In: Knijn, T. \& Komter, A. (Hrsg.), Solidarity between the sexes and the generations: Transformations in Europe. Cheltenham, UK: Edward Elgar, S. 111-130.

Kotowska, I. E. (2005). Work and parenthood: Main findings of comparative data analysis and policy implications. Studia Demograficzne, 2, 148, S. 54-73.

Kotowska, I.E. \& Sztanderska, U. (2007). Zmiany demograficzne a zmiany na rynku pracy w Polsce [Demografischer Wandel und Veränderungen auf dem Arbeitsmarkt in Polen]. In: Kotowska, I. E., Sztanderska U. \& Wóycicka, I. (Hrsg.), Aktywność zawodowa i edukacyjna a obowiazki rodzinne w świetle badań empirycznych. Warschau: Scholar Publishing, S. 13-46.

Koytcheva, E. (2006). Social-demographic differences of fertility and union formation in Bulgaria before and after the start of the societal transition. Rostock: Universität Rostock (Dissertation).

Kravdal, Ø. (2001). The high fertility of college educated women in Norway. Demographic Research, 5 , S. $187-215$.

Kreyenfeld, M. (2002). Time-squeeze, partner effect or self-selection? An investigation into the positive effect of women's education on second birth risks in West Germany. Demographic Research, 7, 2, S. 15-48.

Kreyenfeld, M. (2008). Das zweite Kind in Ostdeutschland: Aufschub oder Verzicht? In: Cassens, I., Luy, M. \& Scholz, R. (Hrsg.), Die Bevölkerung in Ost- und Westdeutschland: Demografische, gesellschaftliche und wirtschaftliche Entwicklungen seit der Wende. Wiesbaden: VS Verlag für Sozialwissenschaften, S. 100-123.

Kreyenfeld, M. \& Zabel, Z. (2005). Female education and the second child: Great Britain and Western Germany compared. Zeitschrift für Wirtschafts- und Sozialwissenschaften/Schmollers Jahrbuch, 125, S. 145-156.

Lappegård, T. \& Rønsen, M. (2005). The multifaceted impact of education on entry into motherhood. European Journal of Population, 21, S. 31-49.

Liefbroer, A. C. \& Corijn, M. (1999). Who, what, where, and when? Specifying the impact of educational attainment and labour force participation on family formation. European Journal of Population, 15, S. 45-75.

Lutz, W., Skirbekk, V. \& Testa, M. R. (2006). The low-fertility trap hypothesis: Forces that may lead to further postponement and fewer births in Europe. Vienna Yearbook of Population Research 2006, S. $167-92$.

Matysiak, A. (2005). The sharing of professional and household duties between Polish couples: Preferences and actual choices. Studia Demograficzne, 1, 147, S. 122-154. 
Matysiak, A. (2007). Organizacja czasu pracy i opieki [Organisation der Arbeitszeit und Pflege]. In: Kotowska, I. E., Sztanderska, U. \& Wóycicka, I. (Hrsg.), Aktywność zawodowa i edukacyjna a obowiazki rodzinne w świetle badań empirycznych. Warschau: Scholar Publishing: 345-80.

Matysiak, A. (2009a). Employment first, then childbearing: Womens strategy in post-socialist Poland. Population Studies: A Journal of Demography, 63, 3, S. 253-276.

Matysiak, A. (2009b). On the interdependencies between fertility and women's labour force participation. Warschau: Warsaw School of Economics (Dissertation).

Matysiak, A. \& S. Steinmetz, S. (2006). Who follows whom? Female employment patterns in West Germany, East Germany and Poland. Mannheim: Mannheimer Zentrum für Europäische Sozialforschung (Arbeitspapiere - Mannheimer Zentrum für Europäische Sozialforschung 94).

Matysiak, A. \& Steinmetz,. S. (2008). Finding their way? Female employment patterns in West Germany, East Germany and Poland. European Sociological Review, 24, 3, S. 331-345.

Matysiak, A. \& Vignoli, D. (2009). Finding the ,right moment" for the first baby to come: A comparison between Italy and Poland. Rostock: Max Planck Institute for Demographic Research (MPIDR Working Paper, WP-2009-011).

Mincer, J. (1974). Schooling, experience and earnings. New York, London: Columbia University Press.

Mincer, J. \& Ofek, H. (1982). Interrupted work careers: Depreciation and restoration of human capital. The Journal of Human Resources, 17, 1, S. 3-24.

Mincer, J. \& Polachek, S. (1974). Family investments in human capital: Earnings of women. The Journal of Political Economy, 82, 2, S. 76-108.

Mishtal, J. Z. (2009). Understanding low fertility in Poland: Demographic consequences of gendered discrimination in employment and post-socialist neoliberal restructuring. Demographic Research, 21, 20, S. 599-626.

Mureşan, C. (2007). Educational attainment and second births in Romania. Rostock: Max Planck Institute for Demographic Research (MPIDR Working Paper WP-2007-028).

Muszyńska, M. (2004). Family models in Europe in the context of women's status. Budapest: Hungarian Central Statistical Office (Working Papers on Population, Family and Welfare, Nr. 6).

Muszyńska, M. (2007). Structural and cultural determinants of fertility in Europe. Warschau: Warsaw School of Economics Publishing.

Neyer, G. R. (2006). Family policies and fertility in Europe: Fertility policies at the intersection of gender policies, employment policies and care policies. Rostock: Max Planck Institute for Demographic Research (MPIDR Working Paper WP-2006-010).

NíBhrolcháin, M. (1986a). The interpretation and role of work-associated accelerated childbearing in post-war Britain. European Journal of Population, 2, 2, S.135-154.

NíBhrolcháin, M. (1986b). Women's paid work and the timing of births. European Journal of Population 2, 1, S. 43-70.

Oláh, L. S. (1996). The impact of public policies on the second-birth tates in Sweden: A gender perspective. Stockholm: University of Stockholm (Stockholm Research Reports in Demography 98).

Oláh, L. S. (2003). Gendering fertility: Second births in Sweden and Hungary. Population Research and Policy Review, 22, 2, S. 171-200.

Perelli-Harris, B. (2008). Family formation in post-Soviet Ukraine: Changing effects of education in a period of rapid social change. Social Forces, 87, 2, S. 767-794.

Potančoková, M., Vaňo, B., Pilinská, V. \& Jurčová, D. (2008). Slovakia: Fertility between tradition and modernity. Demographic Research, 19, 25, S. 973-1018.

Rieck, D. (2006): Transition to second birth - The case of Russia. Rostock: Max Planck Institute for Demographic Research (MPIDR Working Paper WP-2006-036).

Rindfuss, R. R., Guzzo. K. B. \& Morgan, S. P. (2003). The changing institutional context of low fertility. Population Research and Policy Review, 22, S. 411-438.

Schmitt, C. (2007). Familiengründung und Erwerbstätigkeit im Lebenslauf. Aus Politik und Zeitgeschichte, 7, S. 3-8.

Sobotka, T. (2004). Postponement of childbearing and low fertility in Europe. Amsterdam: Dutch University Press. 
Sobotka, T., Lutz, W. \& Philipov, D. (2005). "Missing births": Decomposing the declining number of births in Europe into tempo, quantum, and age structure effects. Wien: Vienna Institute of Demography (European Demographic Research Papers 2/2005).

Sobotka, T., Št’astná, A., Zeman, K., Hamplová. D. \& Kantorová, V. (2008). Czech Republic: A rapid transformation of fertility and family behaviour after the collapse of state socialism. Demographic Research, 19, 14, S. 403-54.

Spéder, Z. \& Kamarás. F. (2008). Hungary: Secular fertility decline with distinct period fluctuations. Demographic Research, 19, 18, S. 599-664

Št'astná, A. (2009). Second births in the Czech Republic. Romanian Journal of Population Studies, 1, S. 109-130.

Sztanderska, U. (2005). Aktywność zawodowa kobiet w Polsce. Jakie szanse? Jakie rezultaty? [Frauenerwerbstätigkeit in Polen. Was sind die Chancen? Was sind die Ergebnisse?]. In: Wóycicka, I. (Hrsg.), Niebieskie księgi: Szanse na wzrost dzietności - jaka polityka rodzinna? Polskie Forum Strategii Lizbońskiej. Gdańsk: Instytut Badań nad Gospodarką Rynkową, S. 43-63.

Testa, M. R. (2006). Childbearing preferences and family issues in Europe. Special Eurobarometer 253/Wave 65.1. Strasbourg: European Commission.

Witte, J. C. \& Wagner, G. G. (1995). Declining fertility in East Germany after unification: A demographic tesponse to socioeconomic change. Population and Development Review. 21, 2, S. 387-397.

Wóycicka, I. (2005). Instrumenty polityki rodzinnej w Polsce na tle doświadczeń międzynarodowych [Instrumente der Familienpolitik in Polen vor dem Hintergrund der internationalen Erfahrungen]. In: Wóycicka, I. (Hrsg.), Niebieskie księgi: Szanse na wzrost dzietności - jaka polityka rodzinna?. Polskie Forum Strategii Lizbońskiej. Gdańsk: Instytut Badań nad Gospodarką Rynkową, S. 79-91.

Eingereicht am/Submitted on: 22.12.2010

Angenommen am/Accepted on: 17.02.2012

Anschrift der Autorin/Address of the author:

Paulina Gałęzewska, M.Sc.

Postgraduate Research Student

Room 2113, Murray Building (58)

Division of Social Statistics and Demography

University of Southampton

Southampton

SO17 1BJ

Vereinigtes Königreich/United Kingdom

Email: pbg1e11@soton.ac.uk 